\title{
Optimization of Brain Conformal Mapping with Landmarks
}

\author{
Yalin Wang ${ }^{1}$, Lok Ming Lui ${ }^{1}$, Tony F. Chan ${ }^{1}$, and Paul M. Thompson ${ }^{2}$ \\ Mathematics Department, UCLA, Los Angeles, CA 90095, USA \\ Lab. of Neuro Imaging, UCLA School of Medicine, Los Angeles, CA 90095, USA \\ \{ylwang, malmlui, chan\}@math.ucla.edu, thompson@loni.ucla.edu
}

\begin{abstract}
To compare and integrate brain data, data from multiple subjects are typically mapped into a canonical space. One method to do this is to conformally map cortical surfaces to the sphere. It is well known that any genus zero Riemann surface can be conformally mapped to a sphere. Therefore, conformal mapping offers a convenient method to parameterize cortical surfaces without angular distortion, generating an orthogonal grid on the cortex that locally preserves the metric. To compare cortical surfaces more effectively, it is advantageous to adjust the conformal parameterizations to match consistent anatomical features across subjects. This matching of cortical patterns improves the alignment of data across subjects, although it is more challenging to create a consistent conformal (orthogonal) parameterization of anatomy across subjects when landmarks are constrained to lie at specific locations in the spherical parameter space. Here we propose a new method, based on a new energy functional, to optimize the conformal parameterization of cortical surfaces by using landmarks. Experimental results on a dataset of 40 brain hemispheres showed that the landmark mismatch energy can be greatly reduced while effectively preserving conformality. The key advantage of this conformal parameterization approach is that any local adjustments of the mapping to match landmarks do not affect the conformality of the mapping significantly. We also examined how the parameterization changes with different weighting factors. As expected, the landmark matching error can be reduced if it is more heavily penalized, but conformality is progressively reduced.
\end{abstract}

\section{Introduction}

An effective way to analyze and compare brain data from multiple subjects is to map them into a canonical space while retaining the original geometric information as far as possible. Surface-based approaches often map cortical surface data to a parameter domain such as a sphere, providing a common coordinate system for data integration 12 . One method is to map the cortical surface conformally to the sphere. Any genus zero Riemann surfaces can be mapped conformally to a sphere, without angular distortion. Therefore, conformal mapping offers a convenient way to parameterize the genus zero cortical surfaces of the brain. To compare cortical surfaces more effectively, it is desirable to adjust the conformal parameterizations to match specific anatomical features on the cortical surfaces 


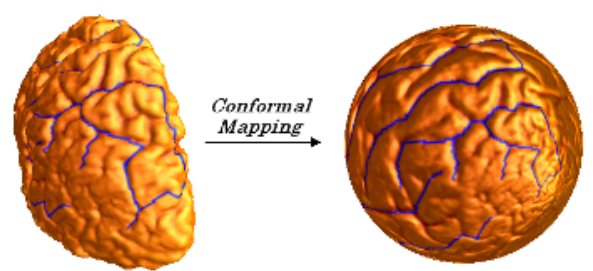

Fig. 1. Manually labeled landmarks on the brain surface. The original surface is on the left. The result of mapping it conformally to a sphere is on the right.

as far as possible (such as sulcal/gyral landmarks in the form of landmark points or 3D curves lying in the surface). Here we refer to these anatomical features as landmarks. Some examples of landmarks are shown in Figure 1.

\subsection{Previous Work}

Several research groups have reported work on brain surface conformal mapping. Hurdal and Stephenson [3] reported a discrete mapping approach that uses circle packing to produce "flattened" images of cortical surfaces on the sphere, the Euclidean plane, or the hyperbolic plane. They obtained maps that are quasi-conformal approximations to classical conformal maps. Haker et al. 4 implemented a finite element approximation for parameterizing brain surfaces via conformal mappings. They represented the Laplace-Beltrami operator as a linear system and solved it for parameterizing brain surfaces via conformal mapping. $\mathrm{Gu}$ et al. [5] proposed a method to find a unique conformal mapping between any two genus zero manifolds by minimizing the harmonic energy of the map. They demonstrated this method by conformally mapping the cortical surface to a sphere.

Optimization of surface diffeomorphisms by landmark matching has been studied intensively. Gu et al. 5] proposed to optimize the conformal parametrization by composing an optimal Möbius transformation so that it minimizes the landmark mismatch energy. The resulting parameterization remains conformal. Glaunés et al. 6] proposed to generate large deformation diffeomorphisms of the sphere onto itself, given the displacements of a finite set of template landmarks. The diffeomorphism obtained can match the geometric features exactly but it is, in general, not a conformal mapping. Leow et al. 7] proposed a level set based approach for matching different types of features, including points and 2D or 3D curves represented as implicit functions. Cortical surfaces were flattened to the unit square. Nine sulcal curves were chosen and were represented by the intersection of two level set functions, and used to constrain the warp of one cortical surface onto another. The resulting transformation was interpolated using a large deformation momentum formulation in the cortical parameter space, generalizing an elastic approach for cortical matching developed in Thompson et al. 8]. Duygu et al. 9] proposed a more automated mapping technique that results in good sulcal alignment across subjects, by combining parametric relaxation, iterative closest point registration and inverse stereographic projection. 


\subsection{Basic Idea}

In this paper, we propose a new method to adjust conformal parameterizations of the cortical surface so that they match consistent anatomical features across subjects. This matching of cortical patterns improves the alignment of data across subjects, e.g., when integrating functional imaging data across subjects, measuring brain changes, or making statistical comparisons in cortical anatomy [10].

Our new method, which is based on a new energy functional, optimizes the conformal parameterization of cortical surfaces by using landmarks. This is done by minimizing the compound energy functional $E_{\text {new }}=E_{\text {harmonic }}+\lambda E_{\text {landmark }}$, where $E_{\text {harmonic }}$ is the harmonic energy of the parameterization and $E_{\text {landmark }}$ is the landmark mismatch energy. We prove theoretically that our proposed $E_{n e w}$ is guaranteed to be decreasing and study the rate of changes of $E_{\text {harmonic }}$ and $E_{\text {landmark }}$. Experimental results show that our algorithm can considerably reduce the landmark mismatch energy while effectively retaining the conformality property. Based on these findings, we argue that the conformal mapping provides an attractive framework to help analyze anatomical shape, and to statistically combine or compare 3D anatomical models across subjects.

\section{Algorithm}

\subsection{Combined Energy Definition}

A diffeomorphism $f: M \rightarrow N$ is a conformal mapping if it preserves the first fundamental form up to a scaling factor (the conformal factor). Mathematically, this means that $d s_{M}^{2}=\lambda f^{*}\left(d s_{N}^{2}\right)$, where $d s_{M}^{2}$ and $d s_{N}^{2}$ are the first fundamental form on $M$ and $N$, respectively and $\lambda$ is the conformal factor. For a diffeomorphism between two genus zero surfaces, a map is conformal if it minimizes the harmonic energy ${ }^{1}, E_{\text {harmonic }}$ [1. Based on this fact, we can compute the conformal mapping by a variational approach, which minimizes the harmonic energy.

Here we propose a new algorithm that optimizes the conformal parameterization using discrete landmarks. This algorithm optimizes the landmark mismatch energy over all degrees of freedom in the reparameterization group. The map obtained can considerably reduce the landmark mismatch energy while retaining conformality as far as possible.

Suppose $C_{1}$ and $C_{2}$ are two cortical surfaces we want to compare. We let $f_{1}: C_{1} \rightarrow S^{2}$ be the conformal parameterization of $C_{1}$ mapping it onto $S^{2}$. We manually label the landmarks on the two cortical surfaces as discrete point sets, as shown in Figure 1. We denote them as $\left\{p_{i} \in C_{1}\right\},\left\{q_{i} \in C_{2}\right\}$, with $p_{i}$ matching $q_{i}$. We proceed to compute a map $f_{2}: C_{2} \rightarrow S^{2}$ from $C_{2}$ to $S^{2}$, which minimizes the harmonic energy as well as minimizing the so-called landmark mismatch energy. The landmark mismatch energy measures the Euclidean distance between

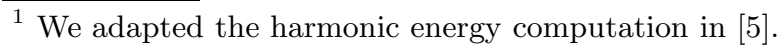


the corresponding landmarks. Alternatively, landmark errors could be computed as geodesic distances with respect to the original surfaces, rather than on the sphere; here we chose to perform distance computations on the sphere. Using our algorithm, the computed map should effectively preserve the conformal property and match the geometric features on the original structures as far as possible.

Let $h: C_{2} \rightarrow S^{2}$ be any homeomorphism from $C_{2}$ onto $S^{2}$. We define the landmark mismatch energy of $h$ as, $\left.E_{\text {landmark }}(h)=1 / 2 \sum_{i=1}^{n} \| h\left(q_{i}\right)\right)-f_{1}\left(p_{i}\right) \|^{2}$. where the norm represents distance on the sphere. By minimizing this energy functional, the Euclidean distance between the corresponding landmarks on the sphere is minimized.

To optimize the conformal parameterization, we propose to find $f_{2}: C_{2} \rightarrow S^{2}$ which minimizes the following new energy functional (instead of the harmonic energy functional), $E_{\text {new }}\left(f_{2}\right)=E_{\text {harmonic }}\left(f_{2}\right)+\lambda E_{\text {landmark }}\left(f_{2}\right)$, where $\lambda$ is a weighting factor (Lagrange multiplier) that balances the two penalty functionals. It controls how much landmark mismatch we want to tolerate. When $\lambda=0$, the new energy functional is just the harmonic energy. When $\lambda$ is large, the landmark mismatch energy can be greatly reduced. But more conformality will be lost (here we regard deviations from conformality to be quantified by the harmonic energy).

Now, let $K$ represent the simplicial realization (triangulation) of the brain surface $C_{2}$, let $u, v$ denote the vertices, and $[u, v]$ denote the edge spanned by $u, v$. Our new energy functional can be written as:

$$
\begin{aligned}
E_{\text {new }}\left(f_{2}\right) & =1 / 2 \sum_{[u, v] \in K} k_{u, v}\left\|f_{2}(u)-f_{2}(v)\right\|^{2}+\lambda / 2 \sum_{i=1}^{n}\left\|f_{2}\left(q_{i}\right)-f_{1}\left(p_{i}\right)\right\|^{2} \\
& \left.=1 / 2 \sum_{[u, v] \in K} k_{u, v}\left\|f_{2}(u)-f_{2}(v)\right\|^{2}+\lambda / 2 \sum_{u \in K} \| f_{2}(u)-L(u)\right) \|^{2} \chi_{M}(u)
\end{aligned}
$$

where $M=\left\{q_{1}, \ldots, q_{n}\right\} ; L\left(q_{i}\right)=p_{i}$ if $u=q_{i} \in M$ and $L(u)=(1,0,0)$ otherwise. The first part of the energy functional is defined as in [5]. Note that by minimizing this energy, we may give up some conformality but the landmark mismatch energy is progressively reduced.

\subsection{Optimization of Combined Energy}

We next formulate a technique to optimize our energy functional. Suppose we would like to compute a mapping $f_{2}$ that minimizes the energy $E_{n e w}\left(f_{2}\right)$. This can be solved easily by steepest descent.

Definition 3.1: Suppose $f \in C^{P L}$, where $C^{P L}$ represent a vector space consists of all piecewise linear functions defined on $K$. We define the Laplacian as follows: $\Delta f(u)=\sum_{[u, v] \in K} k_{u, v}(f(u)-f(v))+\lambda \sum_{u \in K}\left(f_{2}(u)-L(u)\right) \chi_{M}(u)$.

Definition 3.2: Suppose $\vec{f} \in C^{P L}, \vec{f}=\left(f_{0}, f_{1}, f_{2}\right)$, where the $f_{i}$ are piecewise linear. Define the Laplacian of $\vec{f}$ as $\Delta \vec{f}=\left(\Delta f_{0}(u), \Delta f_{1}(u), \Delta f_{2}(u)\right)$.

Now, we know that $f_{2}=\left(f_{20}, f_{21}, f_{22}\right)$ minimizes $E_{\text {new }}\left(f_{2}\right)$ if and only if the tangential component of $\Delta f_{2}(u)=\left(\Delta f_{20}(u), \Delta f_{21}(u), \Delta f_{22}(u)\right)$ vanishes. That is $\Delta\left(f_{2}\right)=\Delta\left(f_{2}\right)^{\perp}$.

In other words, we should have $P_{\vec{n}} \Delta f_{2}(u)=\Delta f_{2}(u)-\left(\Delta f_{2}(u) \cdot \vec{n}\right) \vec{n}=0$. We use a steepest descent algorithm to compute $f_{2}: C_{2} \rightarrow S^{2}: \frac{d f_{2}}{d t}=-P_{\vec{n}} \Delta f_{2}(t)$. 
Algorithm 1. Algorithm to Optimize the Combined Energy $E_{n e w}$ Input (mesh $K$, step length $\delta t$, energy difference threshold $\delta E$ ), output $\left(f_{2}: C_{2} \rightarrow S^{2}\right)$, which minimizes $E$. The computer algorithm proceeds as follows:

1. Given a Gauss map $I: C_{2} \rightarrow S^{2}$. Let $f_{2}=I$, compute $E_{0}=E_{n e w}(I)$

2. For each vertex $v \in K$, compute $P_{\vec{n}} \Delta f_{2}(v)$

3. Update $f_{2}(v)$ by $\delta f_{2}(v)=-P_{\vec{n}} \Delta f_{2}(v) \delta t$

4. Compute energy $E_{n e w}$

5. If $E_{n e w}-E_{0}<\delta E$, return $f_{2}$. Otherwise, assign $E$ to $E_{0}$. Repeat steps 2 to 5 .

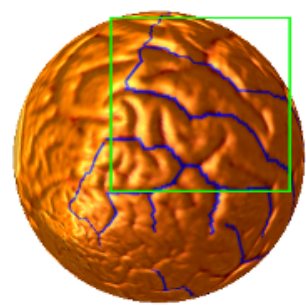

(a)

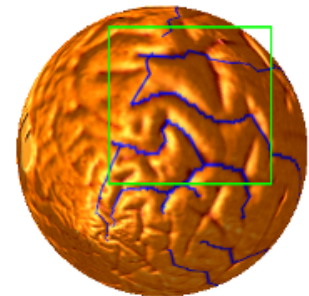

(d)

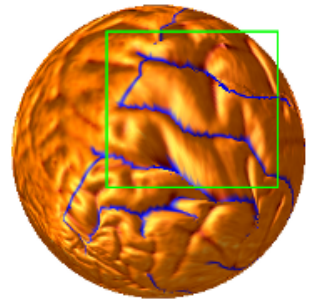

(g)

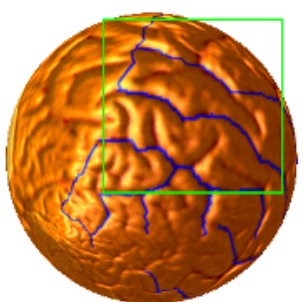

(b)

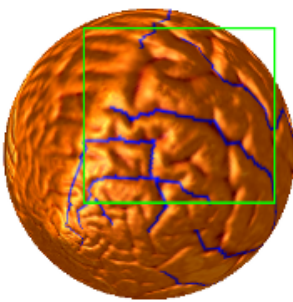

(e)

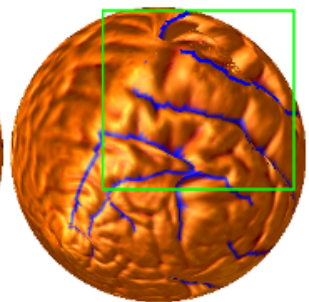

(h).

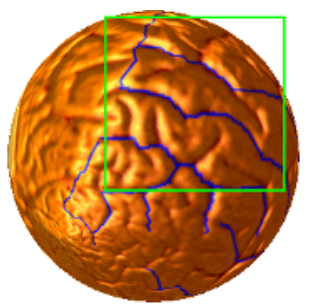

(c)

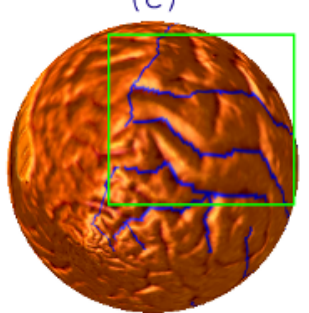

(f)

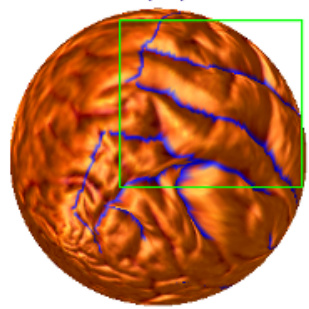

(i)

Fig. 2. In (a), the cortical surface $C_{1}$ (the control) is mapped conformally $(\lambda=0)$ to the sphere. In (d), another cortical surface $C_{2}$ is mapped conformally to the sphere. Note that the sulcal landmarks appear very different from those in (a) (see landmarks in the green square). In (g), the cortical surface $C_{2}$ is mapped to the sphere using our algorithm (with $\lambda=3$ ). Note that the landmarks now closely resemble those in (a) (see landmarks in the green square). (b) and (c) shows the same cortical surface (the control) as in (a). In (e) and (f), two other cortical surfaces are mapped to the spheres. The landmarks again appears very differently. In (h) and (i), the cortical surfaces are mapped to the spheres using our algorithm. The landmarks now closely resemble those of the control. 


\section{Experimental Results}

In our experiment, we tested our algorithm on a set of left hemisphere cortical surfaces generated from brain MRI scans of 40 healthy adult subjects, aged 27.5+/-7.4SD years (16 males, 24 females), scanned at 1.5 T (on a GE Signa scanner). Data and cortical surface landmarks were those generated in a prior paper, Thompson et al. 10 where the extraction and sulcal landmarking procedures are fully detailed. Using this set of 40 hemispheric surfaces, we mapped all surfaces conformally to the sphere and minimized the compound energy matching all subjects to a randomly selected individual subject (alternatively, the surfaces could have been aligned to an average template of curves on the sphere). An important advantage of this approach is that the local adjustments of the mapping to match landmarks do not greatly affect the conformality of the mapping. In Figure 2(a), the cortical surface $C_{1}$ (a control subject) is mapped conformally $(\lambda=0)$ to the sphere. In (d), another cortical surface $C_{2}$ is mapped conformally to the sphere. Note that the sulcal landmarks appear very different from those in (a) (see landmarks in the green square). This means that the geometric features are not well aligned on the sphere unless a further feature-based deformation is applied. In Figure 2(g), we map the cortical surface $C_{2}$ to the sphere with our algorithm, while minimizing the compound energy. This time, the landmarks closely resemble those in (a) (see landmarks in the green square).

In Figure 3, statistics of the angle difference are illustrated. Note that under a conformal mapping, angles between edges on the initial cortical surface should be preserved when these edges are mapped to the sphere. Any differences in angles can be evaluated to determine departures from conformality. Figure 3(a) shows the histogram of the angle difference using the conformal mapping, i.e. after running the algorithm using the conformal energy term only. Figure 2(b) shows the histogram of the angle difference using the compound functional that also penalizes landmark mismatch. Despite the fact that inclusion of landmarks requires more complex mappings, the angular relationships between edges on

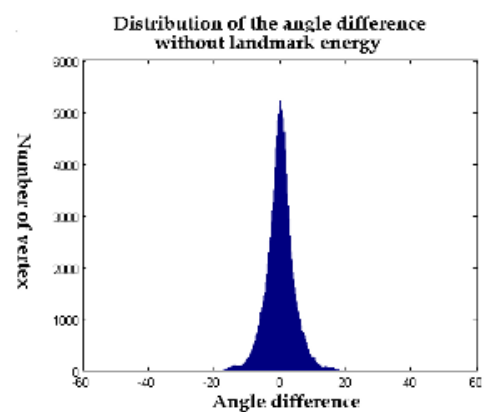

(a)

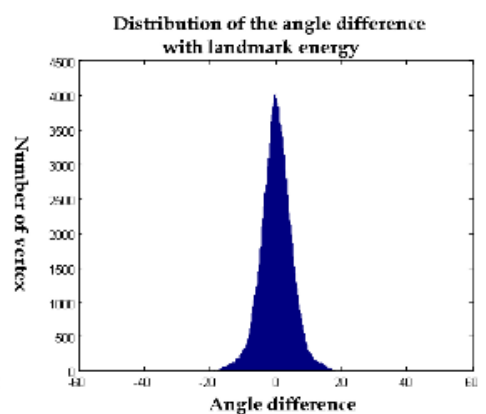

(b)

Fig. 3. Histogram (a) shows the statistics of the angle difference using the conformal mapping. Histogram (b) shows the statistics of the angle difference using our algorithm $(\lambda=3)$. It is observed that the angle is well-preserved. 
Table 1. Numerical data from our experiment. The landmark mismatch energy is greatly reduced while the harmonic energy is only slightly increased. The table also illustrates how the results differ with different values of $\lambda$. The landmark mismatch error can be reduced by increasing $\lambda$, but conformality will increasingly be lost.

\begin{tabular}{cccc}
\hline \hline & $\lambda=3$ & $\lambda=6$ & $\lambda=10$ \\
\hline \hline $\begin{array}{c}E_{\text {harmonic }} \text { of the initial } \\
(\text { conformal) parameterization: }\end{array}$ & 100.6 & 100.6 & 100.6 \\
\hline $\begin{array}{c}\lambda E_{\text {landmark } \text { of the initial (conformal) }} \text { parameterization: } \\
\text { Fitial compound energy }\end{array}$ & 81.2 & & \\
\hline $\begin{array}{c}\text { Initic } \\
\left(E_{\text {harmonic }}+E_{\text {landmark }}\right)\end{array}$ & 181.8 & 263.0 & 371.3 \\
\hline Final $E_{\text {harmonic }}$ & $109.1(\nearrow 8.45 \%)$ & $111.9(\nearrow 11.2 \%)$ & $123.0(\nearrow 22.2 \%)$ \\
\hline Final $\lambda E_{\text {landmark }}$ & $11.2(\searrow 86.2 \%)$ & $13.7(\searrow 91.6 \%)$ & $15.6(\searrow 95.8 \%)$ \\
\hline $\begin{array}{c}\text { Final compound energy } \\
\left(E_{\text {harmonic }}+\lambda E_{\text {landmark }}\right)\end{array}$ & $120.3(\searrow 33.8 \%)$ & $125.6(\searrow 52.2 \%)$ & $138.6(\searrow 62.7 \%)$ \\
\hline
\end{tabular}

the source surface and their images on the sphere are clearly well preserved even after landmark constraints are enforced.

We also tested with other parameter $\lambda$ with different values. Table 1 shows numerical data from the experiment. From the Table, we observe that the landmark mismatch energy is greatly reduced while the harmonic energy is only slightly increased. The table also illustrates how the results differ with different values of $\lambda$. We observe that the landmark mismatch error can be reduced by increasing $\lambda$, but conformality is increasingly lost.

\section{Conclusion and Future Work}

In conclusion, we have developed a new algorithm to compute a map from the cortical surface of the brain to a sphere, which can effectively retain the original geometry by minimizing the landmark mismatch error across different subjects. The development of adjustable landmark weights may be beneficial in computational anatomy. In some applications, such as tracking brain change in an individual over time, in serial images, it makes most sense to place a high priority on landmark correspondence. In other applications, such as the integration of functional brain imaging data across subjects, functional anatomy is not so tightly linked to sulcal landmarks, so it may help to trade landmark error to increase the regularity of the mappings. In the future, we will study the numerical parameters of our algorithm in details to determine how the weighting factor $\lambda$ affects the signal to noise for different neuroimaging applications. We will also compare our algorithm with other existing counterpart quantitatively. Furthermore, more analysis will be done to examine how well the alignment of the sulci/gyri is, such as averaging the maps. 


\section{References}

1. Drury, H., et al. In: Brain Warping. Academic Press (1999) 337-363

2. Fischl, B., et al. In: Human Brain Mapping. Volume 8. (1999) 272-284

3. Hurdal, M.K., Stephenson, K. NeuroImage 23 (2004) S119-S128

4. Haker, S., et al. IEEE TVCG 6 (2000) 181-189

5. Gu, X., et al. IEEE TMI 23 (2004) 949-958

6. Glaunès, J., et al. J. Maths. Imaging and Vision 20 (2004) 179-200

7. Leow, A., et al. NeuroImage 24 (2005) 910-927

8. Thompson, P., et al. Human Brain Mapping 9 (2000) 81-92

9. Tosun, D., et al. Med. Image Anal. 8 (2004) 295-309

10. Thompson, P., et al. NeuroImage 23 (2004) S2-S18

\section{Appendix}

\section{A Energy Is Decreasing}

Claim : With our algorithm, the energy is strictly decreasing.

Proof : Our energy (in continuous form) can be written as: $E(u)=1 / 2 \int\|\nabla u\|^{2}$ $+\lambda \int \delta_{E}\|(u-v)\|^{2}$ where $v$ is the conformal mapping from the control cortical surface to the sphere. Now,

$\left.\frac{d}{d t}\right|_{t=0} E(u+t w)=\int \nabla u \cdot \nabla w+\lambda \int \delta_{E}(u-v) \cdot w=\int \Delta u w+\lambda \int \delta_{E}(u-v) \cdot w$ In our algorithm, the direction $w$ is taken as: $w=-\Delta u-\lambda \delta_{E}(u-v)$. Substituting this into the above equation, we have $\left.\frac{d}{d t}\right|_{t=0} E(u+t w)=-\int\left(\nabla u-(\lambda) \int \delta_{E} \| u-\right.$ $v \|)^{2}<0$. Therefore, the overall energy of the mapping is strictly decreasing, as the iterations proceed.

\section{B Rate of Changes in $E_{\text {harmonic }}$ and $E_{\text {landmark }}$}

To explain why our algorithm can effectively preserve conformalilty while greatly reducing the landmark mismatch energy, we can look at the rate of change of $E_{\text {harmonic }}$ and $E_{\text {landmark }}$. Note that the initial map $u$ we get is almost conformal. Thus, initially $\Delta u$ is very small.

Claim : With our algorithm, the rate of change of $E_{\text {harmonic }}(u)$ is $\mathcal{O}\left(\|\Delta u\|_{\infty}\right)$ and the rate of change of $E_{\text {landmark }}$ is $\lambda^{2} E_{\text {landmark }}(u)+\mathcal{O}\left(\|\Delta u\|_{\infty}\right)$. Here the norm is the supremum norm over the surface.

Proof : Recall that in our algorithm, the direction $w$ is taken as: $w=-\Delta u-$ $\lambda \delta_{E}(u-v)$. Now, the rates of change are:

$$
\begin{aligned}
E_{\text {harmonic }} & =\left|\frac{d}{d t}\right|_{t=0} E_{\text {harmonic }}(u+t w)|=| \int \nabla u \cdot \nabla w|=| \int \Delta u \cdot w \mid \\
& =\left|\int\right|\left|\Delta u\left\|^{2}+\int \delta_{E} \Delta u \cdot(u-v)\left|\leq\|\Delta u\|_{\infty}^{2}+8 \lambda \pi\right| \mid \Delta u\right\|_{\infty}=\mathcal{O}\left(\|\Delta u\|_{\infty}\right)\right. \\
E_{\text {landmark }} & =\left|\frac{d}{d t}\right|_{t=0} E_{\text {landmark }}(u+t w)|=| \int\left(\lambda \delta_{E}\right)^{2}(u-v) \cdot w+\int \delta_{E}(u-v) \cdot \Delta u \mid \\
& \leq \lambda^{2} E_{\text {landmark }}(u)+8 \pi\|\Delta u\|_{\infty}=\lambda^{2} E_{\text {landmark }}(u)+\mathcal{O}\left(\|\Delta u\|_{\infty}\right)
\end{aligned}
$$




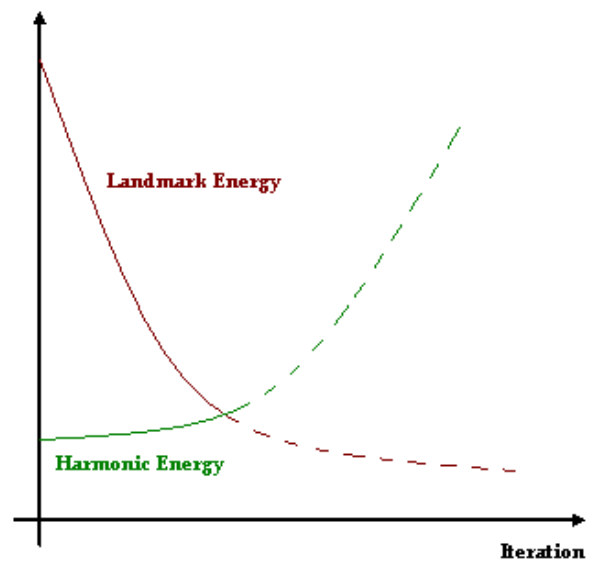

Fig. 4. This figure shows how the harmonic energy and landmark energy change, as the number of iterations increases, using our steepest descent algorithm. Initially, the rate of change of the harmonic energy is small while the rate of change of landmark energy is comparatively large. Note that a Lagrange multiplier governs the weighting of the two energies, so a compromise can be achieved between errors in landmark correspondence and deviations from conformality.

Since initially the map is almost conformal and $\Delta u$ is very small, the change in harmonic energy is very small. Conversely, initially the landmark energy is comparatively large. Since the rate of change of $E_{\text {landmark }}$ is $\lambda^{2} E_{\text {landmark }}(u)+$ $\mathcal{O}\left(\|\Delta u\|_{\infty}\right)$, the change in landmark energy is more significant (see Figure 4 for an illustration). 